\section{Integrative Medicine \\ International}

Integr Med Int 2014;1:185-198

DOI: $10.1159 / 000375532$

Received: December 8, 2014

Accepted after revision: January 26, 2015

Published online: March 19, 2015

This is an Open Access article licensed under the terms of the Creative Commons AttributionNonCommercial 3.0 Unported license (CC BY-NC) (www.karger.com/OA-license), applicable to the online version of the article only. Distribution permitted for non-commercial purposes only

\title{
Identification of Visceral Patterns in Patients with Stress-Related Disorders
}

\author{
Tetsuya Kondo $^{a}$ Shoji Tokunaga ${ }^{c}$ Hideyo Sugahara ${ }^{e}$ \\ Kouichi Yoshimasu $^{b}$ Yoshio Kanemitsu $^{f}$ Chiharu Kubo $^{d}$ \\ ${ }^{a}$ Kansai University of Health Sciences, Kumatori, ${ }^{b}$ Department of Hygiene, School of \\ Medicine, Wakayama Medical University, Wakayama, ${ }^{c}$ Department of Preventive Medicine, \\ Graduate School of Medical Sciences, and dHeadmaster's Office, Kyushu University, \\ eSugahara Tenjin Clinic, and f Department of Psychosomatic Medicine, Fukuoka Dental \\ College, Fukuoka, Japan
}

\author{
Key Words \\ Visceral patterns · Stress-related disorders · Anorexia nervosa $\cdot$ Bulimia nervosa $\cdot$ Eating \\ disorder · Pain disorder - Insomnia · Functional dyspepsia · Irritable bowel syndrome . \\ Hyperventilation syndrome $\cdot$ Bronchial asthma - Dystonia - Psychosomatic disorder . \\ Oriental medicine
}

\section{Abstract}

Background: There is no report on traditional Chinese patterns associated with stress-related disorders such as eating disorder, pain disorder, primary insomnia, functional dyspepsia, irritable bowel syndrome, hyperventilation syndrome, bronchial asthma, and dystonia. Therefore, a large-size case-control study was conducted. Methods: A total of 914 new cases were assessed at a psychosomatic clinic of a tertiary medical care center. The severity of visceral patterns was analyzed according to the main symptoms described and a comprehensive questionnaire. Three patterns of the liver, four of the heart, and one of the kidney as well as dual deficiency of the heart and spleen were assessed. Results: Multiple logistic regression analysis revealed that liver qi depression, liver fire flaming upward, phlegm clouding the pericardium, and dual deficiency of the heart and spleen were associated with bulimia nervosa in females. Liver fire flaming upward and phlegm turbidity were associated with primary insomnia and pain disorder, respectively, in males. An excess pattern was associated with irritable bowel syndrome, while dual deficiency of the heart and spleen was associated with functional dyspepsia in females. On the other hand, the deficiency-excess pattern was reverse in males. Phlegm fire harassing the heart was associated with hyperventilation syndrome in females. Phlegm clouding the pericardium and two kinds of yin deficiency were associated with dystonia in females. Conclusion: Primary insomnia, pain disorder in males, gastrointestinal disorders, and hyperventilation syndrome are each attributed to a single individual pattern. The pattern is different between both sexes. Bulimia nervosa and dystonia are attributed to a few patterns respectively. 


\section{Introduction}

There is well-documented evidence that supports the increasing widespread use of complementary and alternative medicine in the treatment of eating disorders, somatoform disorders, and stress-related medical disease such as functional dyspepsia, irritable bowel syndrome, hyperventilation syndrome, and dystonia [1]. A few traditional Chinese patterns for each disorder are described in the literature. For example, insomnia and psychiatric disorders have been treated with empirical therapy by using Kampo recipes for liver qi depression [2], intense heart fire [3], dual deficiency of the heart and spleen syndrome and yin deficiency [4], as well as phlegm fire harassing the heart, phlegm turbidity, or phlegm clouding the pericardium [5].

However, there is no systematic review on traditional Chinese medicine for these disorders. For example, to the best of our knowledge, only a pilot study on 9 subjects has been reported with respect to eating disorders [6]. Therefore, it remains to be accounted for which pattern is the most important or more important than the others.

Thus, we investigated the association between these disorders and visceral patterns attributed to a large-sized case control study. In the present study, the extent of each visceral pattern was calculated based on the patients' main complaints and their answers to a systematic questionnaire.

\section{Materials and Methods}

\section{Subjects}

This study included 914 outpatients who, for the first time, visited the Department of Psychosomatic Medicine at the Kyushu University Hospital (a tertiary medical care center) between June 2000 and March 2001. Approximately half of them were walk-in patients, and the remaining were referrals. All patients provided written informed consent to participate in the study. The Research Ethics Committee of the Graduate School of Medical Sciences, Kyushu University, approved the protocol of the present survey.

\section{Diagnoses}

After preexamination by a physician specialized in psychosomatic medicine, the patients were assigned to the attending physicians and underwent a thorough checkup. Then, a diagnosis was made by the attending physician according to the DSM-IV. All physicians were also familiar with clinical psychiatry, since they routinely examine patients with mood, anxiety, somatoform, and eating disorders.

\section{Identification of Visceral Patterns}

When associated with these disorders, yin deficiency is categorized into three forms: liver, heart, and kidney yin deficiency. Therefore, it was investigated whether each symptom corresponding to liver qi depression, liver fire flaming upward, dual deficiency of the heart and spleen, intense heart fire, phlegm turbidity, phlegm fire harassing the heart, heart yin deficiency, liver yin deficiency, or kidney yin deficiency was mentioned the patients' chief complaints or their answers to the questionnaires.

Since there are no reports on a systematic scoring system according to traditional Chinese medicine, the scoring for the patterns was conducted provisionally as follows. At the preliminary examination, all patients were asked to describe their main symptoms (up to three complaints). They were also asked to complete the Kyudai Medical Index (KMI) questionnaire. This is a modification of the Cornell Medical Index - Health Questionnaire [7] and 
consists of 103 yes-or-no questions, which include 2 comprehensive questions as well as 2 questions on past history, 2 on family history, 50 on psychiatric symptoms, and 47 on somatic symptoms. The symptom that was described as a chief complaint was considered as 1 point for each visceral pattern. Symptoms that were not described as a chief complaint but were mentioned in the KMI were regarded as $1 / 2$ point for each visceral pattern, since these symptoms can be regarded as relatively minor. The correspondence between the symptoms described as chief complaints or featured in the KMI and each visceral pattern is shown in Appendix 1. The KMI question numbers of the symptoms included in the KMI are also listed. The visceral pattern values were expressed as percentages of the full marks. The World Health Organization (WHO) international standard terminologies on traditional medicine in the Western Pacific region were referred to for all traditional Chinese medicine terminologies [8].

\section{Statistics}

Subjects with organic neurological disorders, endocrine disorders, neoplasms, vascular disorders, infections, degenerative diseases, or traumatism were excluded from the analyses. If a diagnosis could not be identified, the subject was excluded from the study. Other exclusion criteria included failure of the patient to complete the KMI (more than 21 questions unanswered) or to describe the main complaints. Thus, a total of 579 subjects were included. All analyses were performed using Stata version 10.1 (Stata Corp., College Station, Tex., USA). Welch's t test was used to compare the mean continuous variables of the two groups. Multiple logistic regression analyses were performed to estimate the association between the prevalence of occidental diagnoses and each visceral pattern adjusted for age $(<40$ or $>40$ years old). If any case numbers were zero, logistic regression analyses were performed without adjusting for age. A two-sided $p$ value of 0.05 was considered statistically significant.

\section{Results}

\section{Demographic and Occidental Status}

The demographic and occidental aspects of the subjects are shown in table 1 . The continuous variables are shown as means \pm SD. The numbers and percentages of patients with each disorder are also provided. The mean age of the subjects was 36.4 years, with no significant difference between the sexes. The prevalence of eating disorders, somatoform disorders, and irritable bowel syndrome was 10.9, 7.6 and 6.6\%, respectively. The total number of patients with subclassified diseases did not equal the number in each corresponding major division, owing to overlapping. There were some overlaps among psychiatric disorders diagnosed according to DSM-IV and psychosomatic disorders according to the dependent criteria for each disorder. Autonomic ataxia is not an entity defined by DSM-IV. For patients with indefinite complaints, a somatoform disorder is diagnosed according to DSM-IV if psychogenesis or social dysfunction is apparent. Otherwise, autonomic ataxia is diagnosed if objective autonomic dysfunction such as orthostatic hypotension, respiratory arrhythmia or a decrease in the coefficient of variation of RR interval is apparent. Thus, autonomic ataxia was separated from somatoform disorders.

It has been reported that the KMI is able to distinguish healthy controls from patients with neurotic disorders and those with psychosomatic disorders [7]. In this report, the category B question scores were the highest in the subjects with neurosis. The scores in the subjects with psychosomatic disorders were slightly lower than in those with neurosis and considerably higher than in normal subjects [7]. In the present study, the average score for category B questions (i.e., questions assessing psychiatric symptoms) in males aged $<40$ years was 23.1 out of 
Table 1. Demographic data on the study subjects

\begin{tabular}{|c|c|c|c|c|c|}
\hline & \multicolumn{2}{|c|}{ Females $(n=364)$} & \multicolumn{2}{|c|}{ Males $(n=215)$} & \multirow{2}{*}{$\begin{array}{l}\text { Total } \\
(n=579)\end{array}$} \\
\hline & $\begin{array}{l}<40 \text { years old } \\
(\mathrm{n}=229)\end{array}$ & $\begin{array}{l}>40 \text { years old } \\
(\mathrm{n}=135)\end{array}$ & $\begin{array}{l}<40 \text { years old } \\
(n=138)\end{array}$ & $\begin{array}{l}>40 \text { years old } \\
(n=77)\end{array}$ & \\
\hline Age, years & $25.0 \pm 6.7$ & $55.9 \pm 10.1$ & $25.5 \pm 7.5$ & $55.4 \pm 10.9$ & $36.4 \pm 16.9$ \\
\hline SDS & $52.8 \pm 9.8$ & $50.5 \pm 10.4$ & $48.7 \pm 9.8$ & $47.6 \pm 11.3$ & $50.6 \pm 10.3$ \\
\hline STAI-I & $55.6 \pm 11.4$ & $55 \pm 10.9$ & $53.9 \pm 11.7$ & $53.1 \pm 12.1$ & $54.8 \pm 11.5$ \\
\hline STAI-II & $57.5 \pm 12$ & $53.4 \pm 10.9$ & $54.6 \pm 11.3$ & $52.7 \pm 14$ & $55.3 \pm 12.0$ \\
\hline KMI category B questions & $24.5 \pm 9.7$ & $21.8 \pm 11.0$ & $23.1 \pm 10.5$ & $22.7 \pm 10.9$ & $23.3 \pm 10.4$ \\
\hline KMI category $\mathrm{C}$ questions & $17.3 \pm 8.1$ & $15.9 \pm 8.7$ & $14.3 \pm 7.5$ & $16.0 \pm 7.7$ & $16.1 \pm 8.2$ \\
\hline Mood disorders & $112(48.9)$ & $93(68.9)$ & $68(49.3)$ & $41(53.2)$ & $314(54.2)$ \\
\hline Major depression & $85(41.7)$ & $73(62.9)$ & $45(38.8)$ & $34(48.6)$ & $237(46.8)$ \\
\hline Dysthymic disorder & $10(4.4)$ & $13(9.6)$ & $4(2.9)$ & $4(5.2)$ & $31(5.4)$ \\
\hline \multicolumn{6}{|l|}{ Depressive disorder not otherwise } \\
\hline specified & $26(11.4)$ & $16(11.9)$ & $22(15.9)$ & $7(9.1)$ & $71(12.3)$ \\
\hline Anxiety disorders & $46(20.1)$ & $25(18.5)$ & $34(24.6)$ & $10(13.0)$ & $115(19.9)$ \\
\hline Generalized anxiety disorder & $15(6.6)$ & $20(14.8)$ & $15(10.9)$ & $7(9.1)$ & $57(9.8)$ \\
\hline Specific phobia & $3(1.3)$ & $0(0.0)$ & $1(0.7)$ & $1(1.3)$ & $5(0.9)$ \\
\hline Panic disorder & $19(8.3)$ & $5(3.7)$ & $8(5.8)$ & $3(3.9)$ & $35(6.0)$ \\
\hline Posttraumatic stress disorder & $1(0.4)$ & $0(0.0)$ & $2(1.4)$ & $0(0.0)$ & $3(0.5)$ \\
\hline Social anxiety disorder & $4(1.7)$ & $0(0.0)$ & $8(5.8)$ & $0(0.0)$ & $12(2.1)$ \\
\hline Obsessive compulsive disorder & $5(2.2)$ & $0(0.0)$ & $1(0.7)$ & $0(0.0)$ & $6(1.0)$ \\
\hline Eating disorders & $56(24.5)$ & $1(0.7)$ & $5(3.6)$ & $1(1.3)$ & $63(10.9)$ \\
\hline Anorexia nervosa & $16(7.0)$ & $1(0.7)$ & $2(1.4)$ & $1(1.3)$ & $20(3.5)$ \\
\hline Bulimia nervosa & $27(11.8)$ & $0(0.0)$ & $1(0.7)$ & $0(0.0)$ & $28(4.8)$ \\
\hline Not otherwise specified & $13(5.7)$ & $0(0.0)$ & $2(1.4)$ & $0(0.0)$ & $15(2.6)$ \\
\hline Somatoform disorders & $13(5.7)$ & $15(11.1)$ & $6(4.3)$ & $10(13.0)$ & $44(7.6)$ \\
\hline Conversion disorder & $2(0.9)$ & $2(1.5)$ & $0(0.0)$ & $0(0.0)$ & $4(0.7)$ \\
\hline Hypochondriasis & $0(0.0)$ & $3(2.2)$ & $1(0.7)$ & $2(2.6)$ & $6(1.0)$ \\
\hline Somatization disorder & $0(0.0)$ & $1(0.7)$ & $0(0.0)$ & $0(0.0)$ & $1(0.2)$ \\
\hline Pain disorder & $9(3.9)$ & $6(4.4)$ & $3(2.2)$ & $7(9.1)$ & $25(4.3)$ \\
\hline Not otherwise specified & $2(0.9)$ & $2(1.5)$ & $1(0.7)$ & $1(1.3)$ & $6(1.0)$ \\
\hline Irritable bowel syndrome & $15(6.6)$ & $9(6.7)$ & $8(5.8)$ & $6(7.8)$ & $38(6.6)$ \\
\hline Functional dyspepsia & $15(6.6)$ & $0(0.0)$ & $8(5.8)$ & $1(1.3)$ & $24(4.1)$ \\
\hline Primary insomnia & $5(2.2)$ & $2(1.5)$ & $6(4.3)$ & $7(9.1)$ & $20(3.5)$ \\
\hline Hypertension & $4(1.7)$ & $4(3.0)$ & $3(2.2)$ & $2(2.6)$ & $13(2.2)$ \\
\hline Hyperventilation syndrome & $8(3.5)$ & $2(1.5)$ & $2(1.4)$ & $0(0.0)$ & $12(2.1)$ \\
\hline Bronchial asthma & $5(2.2)$ & $3(2.2)$ & $1(0.7)$ & $0(0.0)$ & $9(1.6)$ \\
\hline Dystonia & $2(0.9)$ & $4(3.0)$ & $1(0.7)$ & $1(1.3)$ & $8(1.4)$ \\
\hline Schizophrenia & $2(0.9)$ & $2(1.5)$ & $0(0.0)$ & $4(5.2)$ & $8(1.4)$ \\
\hline Chronic fatigue syndrome & $0(0.0)$ & $2(1.5)$ & $1(0.7)$ & $0(0.0)$ & $3(0.5)$ \\
\hline Dissociative disorder & $2(0.9)$ & $0(0.0)$ & $0(0.0)$ & $0(0.0)$ & $2(0.3)$ \\
\hline
\end{tabular}

Values are presented as means \pm SD or n (\%). SDS = Self-rating Depression Scale; STAI = Stait-Trait Anxiety Inventory.

50 points, which was higher than the average reported previously in normal subjects ( 9.5 out of 50 points) and in subjects with psychosomatic disorders (18.8 out of 50 points) but lower than in those with neurosis ( 24.4 out of 50 points). The average score for category C questions in this group was 14.3 points, which was lower than that of patients with psychosomatic disorders (14.8 points) and neurosis (23.1 out of 50 points) [7]. Thus, this group was regarded as psychosomatic as well as neurotic. However, in the other three groups, the average scores for category B questions were all higher than those of psychosomatic disorders and close to those of neurosis. The average scores for category $\mathrm{C}$ questions were higher than those of neurosis. Thus, these groups were regarded as neurotic rather than psychosomatic. 
Table 2. Visceral pattern scores

\begin{tabular}{|c|c|c|c|c|c|}
\hline & \multicolumn{2}{|c|}{ Males $(n=215)$} & \multicolumn{2}{|c|}{ Females $(n=364)$} & \multirow{2}{*}{$\begin{array}{l}\text { Sexual } \\
\text { difference, } \\
\mathrm{p}\end{array}$} \\
\hline & mean $\pm \mathrm{SD}$ & $\min -\max$ & mean $\pm \mathrm{SD}$ & $\min -\max$ & \\
\hline Liver qi depression & $11.1 \pm 5.6$ & $0.0-27.7$ & $11.8 \pm 6.2$ & $0.0-29.8$ & 0.13 \\
\hline Liver fire flaming upward & $16.6 \pm 9.1$ & $0.0-41.7$ & $17.1 \pm 9.4$ & $0.0-45.8$ & 0.58 \\
\hline Intense heart fire & $13.9 \pm 9.0$ & $0.0-38.1$ & $13.8 \pm 8.8$ & $0.0-38.1$ & 0.91 \\
\hline Phlegm fire harassing the heart & $13.5 \pm 7.0$ & $0.0-37.1$ & $14.8 \pm 7.8$ & $0.0-34.3$ & 0.04 \\
\hline Phlegm turbidity & $14.7 \pm 10.8$ & $0.0-46.2$ & $15.3 \pm 10.6$ & $0.0-53.8$ & 0.50 \\
\hline Phlegm clouding the pericardium & $24.8 \pm 15.2$ & $0.0-60.0$ & $25.2 \pm 13.9$ & $0.0-55.0$ & 0.78 \\
\hline Dual deficiency of the heart and spleen & $19.6 \pm 10.0$ & $0.0-50.0$ & $22.5 \pm 10.1$ & $0.0-47.6$ & $<0.005$ \\
\hline Liver yin deficiency & $13.8 \pm 7.5$ & $0.0-35.5$ & $14.0 \pm 7.3$ & $0.0-35.5$ & 0.75 \\
\hline Heart yin deficiency & $17.1 \pm 9.2$ & $0.0-39.0$ & $17.2 \pm 8.9$ & $0.0-39.0$ & 0.97 \\
\hline Kidney yin deficiency & $11.4 \pm 6.5$ & $0.0-27.8$ & $11.4 \pm 6.3$ & $0.0-30.6$ & 0.94 \\
\hline
\end{tabular}

\section{Sexual Differences in Visceral Patterns}

In order to investigate visceral patterns according to sex, an analysis of covariance was performed. As shown in table 2, dual vacuity of the heart and spleen was significantly higher in females than males. There were no sexual differences in the other visceral patterns.

\section{Visceral Patterns in Eating Disorders}

The association between the disorders and the patterns are shown in table 3.

Although there was no association between eating disorders and visceral patterns in males, liver qi depression was significantly and heart yin deficiency inversely associated with all forms of eating disorders in females.

The visceral patterns were different between anorexia nervosa and bulimia nervosa in females. Liver fire flaming upward was inversely associated, but no patterns were positively associated with anorexia nervosa. On the other hand, liver qi depression, liver fire flaming upward, phlegm clouding the pericardium, and dual deficiency of the heart and spleen were significantly associated with bulimia nervosa. Phlegm fire harassing the heart and kidney yin deficiency were inversely associated with this disorder.

On the other hand, kidney yin deficiency was significantly associated with not otherwise specified eating disorder in females. No other patterns were neither significantly nor inversely associated with this disorder.

\section{Visceral Patterns in Somatoform Disorders}

Although no association between somatoform disorders and visceral patterns was revealed in females, some associations were observed in males. Phlegm turbidity was significantly associated with pain disorder. On the other hand, liver qi depression was inversely associated with all forms of somatoform disorders and pain disorder in males. No association was observed with pain disorder in females or all forms of somatoform disorders in both sexes and patters.

\section{Visceral Patterns in Primary Insomnia}

Liver fire flaming upward was significantly associated and phlegm-fire harassing the heart was inversely associated with primary insomnia in males, while no visceral pattern was significantly or inversely associated in females. 
Table 3. Associations between disorders and patterns

\begin{tabular}{|c|c|c|c|c|}
\hline \multirow[t]{2}{*}{ Variables } & \multicolumn{2}{|l|}{ Females } & \multicolumn{2}{|l|}{ Males } \\
\hline & odds ratio $(95 \% \mathrm{CI})$ & $\mathrm{p}$ & odds ratio $(95 \% \mathrm{CI})$ & $\mathrm{p}$ \\
\hline \multicolumn{5}{|l|}{ Eating disorders } \\
\hline \multicolumn{5}{|l|}{ All forms } \\
\hline Liver qi depression & $2.98(1.39-6.38)$ & $<0.01$ & $0.40(0.03-5.83)$ & 0.50 \\
\hline Liver fire flaming upward & $1.10(0.59-2.05)$ & 0.75 & $0.25(0.04-1.78)$ & 0.17 \\
\hline Dual deficiency of the heart and spleen & $1.29(0.74-2.25)$ & 0.37 & $2.89(0.43-19.49)$ & 0.27 \\
\hline Intense heart fire & $1.05(0.50-2.22)$ & 0.89 & $1.11(0.15-8.04)$ & 0.91 \\
\hline Phlegm turbidity & $0.88(0.53-1.47)$ & 0.63 & $0.65(0.17-2.47)$ & 0.52 \\
\hline Phlegm fire harassing the heart & $0.43(0.18-1.04)$ & 0.06 & $2.03(0.21-19.51)$ & 0.54 \\
\hline Phlegm clouding the pericardium & $1.23(0.82-1.84)$ & 0.31 & $0.90(0.30-2.64)$ & 0.84 \\
\hline Liver yin deficiency & $0.90(0.37-2.14)$ & 0.80 & $0.85(0.05-14.25)$ & 0.91 \\
\hline Heart yin deficiency & $0.38(0.18-0.80)$ & 0.01 & $1.10(0.14-8.58)$ & 0.93 \\
\hline Kidney yin deficiency & $1.20(0.54-2.65)$ & 0.66 & $3.59(0.30-42.68)$ & 0.31 \\
\hline \multicolumn{5}{|l|}{ Anorexia nervosa } \\
\hline Liver qi depression & $1.00(0.23-4.26)$ & 1.00 & - & - \\
\hline Liver fire flaming upward & $0.30(0.10-0.96)$ & 0.04 & - & - \\
\hline Dual deficiency of the heart and spleen & $0.76(0.27-2.14)$ & 0.61 & - & - \\
\hline Intense heart fire & $1.07(0.26-4.43)$ & 0.92 & - & - \\
\hline Phlegm turbidity & $0.88(0.37-2.12)$ & 0.78 & - & - \\
\hline Phlegm fire harassing the heart & $1.19(0.26-5.39)$ & 0.83 & - & - \\
\hline Phlegm clouding the pericardium & $0.96(0.46-2.00)$ & 0.91 & - & - \\
\hline Liver yin deficiency & $2.70(0.62-11.73)$ & 0.18 & - & - \\
\hline Heart yin deficiency & $0.46(0.11-1.95)$ & 0.29 & - & - \\
\hline Kidney yin deficiency & $2.42(0.59-9.96)$ & 0.22 & - & - \\
\hline \multicolumn{5}{|l|}{ Bulimia nervosa } \\
\hline Liver qi depression & $6.35(1.99-20.22)$ & $<0.01$ & - & - \\
\hline Liver fire flaming upward & $4.63(1.65-13.00)$ & $<0.01$ & - & - \\
\hline Dual deficiency of the heart and spleen & $2.49(1.07-5.80)$ & 0.03 & - & - \\
\hline Intense heart fire & $0.80(0.28-2.30)$ & 0.69 & - & - \\
\hline Phlegm turbidity & $0.44(0.18-1.10)$ & 0.08 & - & - \\
\hline Phlegm fire harassing the heart & $0.19(0.04-0.80)$ & 0.02 & - & - \\
\hline Phlegm clouding the pericardium & $2.06(1.10-3.84)$ & 0.02 & - & - \\
\hline Liver yin deficiency & $0.42(0.11-1.63)$ & 0.21 & - & - \\
\hline Heart yin deficiency & $0.34(0.12-1.01)$ & 0.05 & - & - \\
\hline Kidney yin deficiency & $0.21(0.06-0.80)$ & 0.02 & - & - \\
\hline \multicolumn{5}{|l|}{ Not otherwise specified } \\
\hline Liver qi depression & $2.77(0.67-11.47)$ & 0.16 & - & - \\
\hline Liver fire flaming upward & $0.59(0.17-2.01)$ & 0.40 & - & - \\
\hline Dual deficiency of the heart and spleen & $0.95(0.31-2.93)$ & 0.92 & - & - \\
\hline Intense heart fire & $1.24(0.28-5.53)$ & 0.78 & - & - \\
\hline Phlegm turbidity & $1.62(0.63-4.19)$ & 0.32 & - & - \\
\hline Phlegm fire harassing the heart & $0.57(0.10-3.30)$ & 0.53 & - & - \\
\hline Phlegm clouding the pericardium & $0.88(0.39-1.98)$ & 0.76 & - & - \\
\hline Liver yin deficiency & $0.82(0.15-4.44)$ & 0.82 & - & - \\
\hline Heart yin deficiency & $0.76(0.18-3.25)$ & 0.71 & - & - \\
\hline Kidney yin deficiency & $6.14(1.30-28.92)$ & 0.02 & - & - \\
\hline \multicolumn{5}{|l|}{ Somatoform disorders } \\
\hline \multicolumn{5}{|l|}{ All forms } \\
\hline Liver qi depression & $0.49(0.15-1.60)$ & 0.24 & $0.09(0.01-0.63)$ & 0.02 \\
\hline Liver fire flaming upward & $0.90(0.37-2.20)$ & 0.82 & $1.34(0.43-4.24)$ & 0.62 \\
\hline Dual deficiency of the heart and spleen & $0.79(0.34-1.83)$ & 0.58 & $2.39(0.67-8.48)$ & 0.18 \\
\hline Intense heart fire & $2.60(0.94-7.21)$ & 0.07 & $1.92(0.49-7.45)$ & 0.35 \\
\hline Phlegm turbidity & $0.65(0.31-1.36)$ & 0.25 & $1.31(0.52-3.32)$ & 0.57 \\
\hline Phlegm fire harassing the heart & $1.31(0.44-3.90)$ & 0.63 & $3.02(0.59-15.39)$ & 0.18 \\
\hline Phlegm clouding the pericardium & $0.75(0.42-1.36)$ & 0.35 & $0.81(0.37-1.75)$ & 0.59 \\
\hline
\end{tabular}


Table 3 (continued)

\begin{tabular}{|c|c|c|c|c|}
\hline \multirow[t]{2}{*}{ Variables } & \multicolumn{2}{|l|}{ Females } & \multicolumn{2}{|l|}{ Males } \\
\hline & odds ratio $(95 \% \mathrm{CI})$ & $\mathrm{p}$ & odds ratio $(95 \% \mathrm{CI})$ & $\mathrm{p}$ \\
\hline Liver yin deficiency & $1.65(0.50-5.45)$ & 0.41 & $0.92(0.15-5.60)$ & 0.93 \\
\hline Heart yin deficiency & $0.74(0.27-2.07)$ & 0.57 & $0.57(0.12-2.70)$ & 0.47 \\
\hline Kidney yin deficiency & $2.64(0.90-7.71)$ & 0.08 & $2.17(0.44-10.65)$ & 0.34 \\
\hline \multicolumn{5}{|l|}{ Pain disorder } \\
\hline Liver qi depression & $0.33(0.07-1.62)$ & 0.17 & $0.02(0.00-0.50)$ & 0.02 \\
\hline Liver fire flaming upward & $0.85(0.26-2.76)$ & 0.78 & $2.99(0.52-17.08)$ & 0.22 \\
\hline Dual deficiency of the heart and spleen & $0.58(0.19-1.74)$ & 0.33 & $2.39(0.52-10.90)$ & 0.26 \\
\hline Intense heart fire & $2.27(0.63-8.19)$ & 0.21 & $1.81(0.29-11.24)$ & 0.53 \\
\hline Phlegm turbidity & $0.80(0.32-1.98)$ & 0.62 & $3.89(1.10-13.80)$ & 0.04 \\
\hline Phlegm fire harassing the heart & $1.40(0.35-5.63)$ & 0.63 & $2.11(0.29-15.37)$ & 0.46 \\
\hline Phlegm clouding the pericardium & $1.04(0.49-2.21)$ & 0.92 & $0.61(0.23-1.64)$ & 0.33 \\
\hline Liver yin deficiency & $2.21(0.50-9.72)$ & 0.29 & $1.67(0.16-17.12)$ & 0.67 \\
\hline Heart yin deficiency & $1.25(0.34-4.59)$ & 0.74 & $1.83(0.23-14.33)$ & 0.56 \\
\hline Kidney yin deficiency & $2.26(0.59-8.72)$ & 0.24 & $2.35(0.34-16.29)$ & 0.39 \\
\hline \multicolumn{5}{|l|}{ Primary insomnia } \\
\hline Liver qi depression & $0.21(0.02-1.92)$ & 0.17 & $0.60(0.07-5.27)$ & 0.65 \\
\hline Liver fire flaming upward & $0.90(0.17-4.91)$ & 0.91 & $12.80(2.39-68.60)$ & $<0.01$ \\
\hline Dual deficiency of the heart and spleen & $1.57(0.36-6.89)$ & 0.55 & $1.29(0.28-6.06)$ & 0.74 \\
\hline Intense heart fire & $3.98(0.51-30.83)$ & 0.19 & $1.84(0.39-8.78)$ & 0.44 \\
\hline Phlegm turbidity & $1.31(0.35-4.83)$ & 0.69 & $1.92(0.63-5.85)$ & 0.25 \\
\hline Phlegm fire harassing the heart & $0.31(0.03-3.75)$ & 0.36 & $0.03(0.00-0.35)$ & $<0.01$ \\
\hline Phlegm clouding the pericardium & $1.79(0.63-5.09)$ & 0.28 & $0.91(0.37-2.28)$ & 0.84 \\
\hline Liver yin deficiency & $3.44(0.42-28.25)$ & 0.25 & $2.04(0.32-12.92)$ & 0.45 \\
\hline Heart yin deficiency & $0.42(0.06-3.11)$ & 0.40 & $0.75(0.14-4.02)$ & 0.74 \\
\hline Kidney yin deficiency & $0.28(0.02-3.10)$ & 0.30 & $0.28(0.04-1.94)$ & 0.20 \\
\hline \multicolumn{5}{|l|}{ Functional dyspepsia } \\
\hline Liver qi depression & $4.18(0.95-18.45)$ & 0.06 & $16.74(1.97-142.60)$ & $<0.01$ \\
\hline Liver fire flaming upward & $1.87(0.59-5.90)$ & 0.29 & $0.16(0.02-1.63)$ & 0.12 \\
\hline Dual deficiency of the heart and spleen & $4.24(1.31-13.77)$ & 0.02 & $1.58(0.34-7.38)$ & 0.56 \\
\hline Intense heart fire & $2.32(0.50-10.75)$ & 0.28 & $0.39(0.05-3.14)$ & 0.37 \\
\hline Phlegm turbidity & $1.94(0.69-5.51)$ & 0.21 & $0.47(0.12-1.82)$ & 0.27 \\
\hline Phlegm fire harassing the heart & $0.15(0.02-1.10)$ & 0.06 & $3.94(0.47-33.15)$ & 0.21 \\
\hline Phlegm clouding the pericardium & $0.53(0.23-1.21)$ & 0.13 & $0.68(0.24-1.94)$ & 0.47 \\
\hline Liver yin deficiency & $0.35(0.06-2.09)$ & 0.25 & $0.76(0.09-6.58)$ & 0.81 \\
\hline Heart yin deficiency & $0.10(0.02-0.55)$ & $<0.01$ & $0.50(0.06-4.45)$ & 0.54 \\
\hline Kidney yin deficiency & $1.81(0.36-9.05)$ & 0.47 & $1.13(0.12-10.64)$ & 0.92 \\
\hline \multicolumn{5}{|l|}{ Irritable bowel syndrome } \\
\hline Liver qi depression & $3.40(1.01-11.42)$ & 0.05 & $3.89(0.84-17.99)$ & 0.08 \\
\hline Liver fire flaming upward & $1.38(0.48-3.95)$ & 0.55 & $0.44(0.09-2.22)$ & 0.32 \\
\hline Dual deficiency of the heart and spleen & $1.76(0.69-4.50)$ & 0.24 & $6.58(1.88-23.00)$ & $<0.01$ \\
\hline Intense heart fire & $4.17(1.13-15.37)$ & 0.03 & $4.31(1.01-18.37)$ & 0.05 \\
\hline Phlegm turbidity & $1.61(0.69-3.76)$ & 0.27 & $0.75(0.29-1.93)$ & 0.55 \\
\hline Phlegm fire harassing the heart & $0.43(0.09-2.07)$ & 0.29 & $0.15(0.02-1.10)$ & 0.06 \\
\hline Phlegm clouding the pericardium & $0.66(0.33-1.32)$ & 0.24 & $0.83(0.39-1.76)$ & 0.62 \\
\hline Liver yin deficiency & $0.17(0.04-0.77)$ & 0.02 & $1.06(0.20-5.47)$ & 0.95 \\
\hline Heart yin deficiency & $0.29(0.08-1.03)$ & 0.05 & $0.12(0.03-0.56)$ & $<0.01$ \\
\hline Kidney yin deficiency & $0.85(0.21-3.46)$ & 0.82 & $1.15(0.18-7.27)$ & 0.88 \\
\hline \multicolumn{5}{|l|}{ Hyperventilation syndrome } \\
\hline Liver qi depression & $3.36(0.54-20.87)$ & 0.19 & - & - \\
\hline Liver fire flaming upward & $0.65(0.15-2.76)$ & 0.56 & - & - \\
\hline Dual deficiency of the heart and spleen & $0.37(0.09-1.52)$ & 0.17 & - & - \\
\hline Intense heart fire & $0.41(0.08-2.08)$ & 0.28 & - & - \\
\hline
\end{tabular}


Table 3 (continued)

\begin{tabular}{|c|c|c|c|c|}
\hline \multirow[t]{2}{*}{ Variables } & \multicolumn{2}{|l|}{ Females } & \multicolumn{2}{|l|}{ Males } \\
\hline & odds ratio $(95 \% \mathrm{CI})$ & $\mathrm{p}$ & odds ratio $(95 \% \mathrm{CI})$ & $\mathrm{p}$ \\
\hline Phlegm turbidity & $0.59(0.20-1.75)$ & 0.34 & - & - \\
\hline Phlegm fire harassing the heart & $39.76(6.00-263.30)$ & $<0.001$ & - & - \\
\hline Phlegm clouding the pericardium & $0.65(0.24-1.78)$ & 0.41 & - & - \\
\hline Liver yin deficiency & $0.34(0.04-3.26)$ & 0.35 & - & - \\
\hline Heart yin deficiency & $1.05(0.20-5.55)$ & 0.95 & - & - \\
\hline Kidney yin deficiency & $0.41(0.04-3.89)$ & 0.43 & - & - \\
\hline \multicolumn{5}{|l|}{ Bronchial asthma } \\
\hline Liver qi depression & $1.46(0.14-15.25)$ & 0.75 & - & - \\
\hline Liver fire flaming upward & $0.95(0.18-4.98)$ & 0.95 & - & - \\
\hline Dual deficiency of the heart and spleen & $0.43(0.08-2.22)$ & 0.31 & - & - \\
\hline Intense heart fire & $0.43(0.06-3.14)$ & 0.41 & - & - \\
\hline Phlegm turbidity & $0.18(0.03-0.94)$ & 0.04 & - & - \\
\hline Phlegm fire harassing the heart & $4.22(0.62-28.77)$ & 0.14 & - & - \\
\hline Phlegm clouding the pericardium & $1.73(0.54-5.48)$ & 0.35 & - & - \\
\hline Liver yin deficiency & $1.28(0.11-15.42)$ & 0.85 & - & - \\
\hline Heart yin deficiency & $0.43(0.06-3.32)$ & 0.42 & - & - \\
\hline Kidney yin deficiency & $1.70(0.20-14.43)$ & 0.63 & - & - \\
\hline \multicolumn{5}{|l|}{ Dystonia } \\
\hline Liver qi depression & $1.05(0.01-212.88)$ & 0.99 & - & - \\
\hline Liver fire flaming upward & $0.14(0.00-5.78)$ & 0.30 & - & - \\
\hline Dual deficiency of the heart and spleen & $0.03(0.00-1.14)$ & 0.06 & - & - \\
\hline Intense heart fire & $99.00(0.76-12822.25)$ & 0.06 & - & - \\
\hline Phlegm turbidity & $0.00(0.00-0.42)$ & 0.02 & - & - \\
\hline Phlegm fire harassing the heart & $0.58(0.03-10.93)$ & 0.71 & - & - \\
\hline Phlegm clouding the pericardium & $24.32(1.11-532.42)$ & 0.04 & - & - \\
\hline Liver yin deficiency & $458.52(1.92-109,233.09)$ & 0.03 & - & - \\
\hline Heart yin deficiency & $0.08(0.00-4.96)$ & 0.23 & - & - \\
\hline Kidney yin deficiency & $210.60(1.72-25,780.90)$ & 0.03 & - & - \\
\hline \multicolumn{5}{|l|}{ Autonomic ataxia } \\
\hline Liver qi depression & $0.45(0.10-1.99)$ & 0.29 & $0.12(0.01-2.28)$ & 0.16 \\
\hline Liver fire flaming upward & $2.34(0.79-6.94)$ & 0.12 & $1.50(0.26-8.55)$ & 0.65 \\
\hline Dual deficiency of the heart and spleen & $1.12(0.41-3.02)$ & 0.83 & $2.47(0.42-14.49)$ & 0.32 \\
\hline Intense heart fire & $0.50(0.14-1.85)$ & 0.30 & $1.45(0.22-9.54)$ & 0.70 \\
\hline Phlegm turbidity & $1.70(0.76-3.82)$ & 0.20 & $2.45(0.73-8.24)$ & 0.15 \\
\hline Phlegm fire harassing the heart & $1.28(0.34-4.79)$ & 0.72 & $0.25(0.02-2.74)$ & 0.26 \\
\hline Phlegm clouding the pericardium & $0.57(0.28-1.15)$ & 0.12 & $0.38(0.13-1.11)$ & 0.08 \\
\hline Liver yin deficiency & $1.78(0.40-8.00)$ & 0.45 & $26.47(2.09-335.42)$ & 0.01 \\
\hline Heart yin deficiency & $2.24(0.63-7.93)$ & 0.21 & $2.66(0.39-18.11)$ & 0.32 \\
\hline Kidney yin deficiency & $0.70(0.17-2.85)$ & 0.62 & $1.18(0.12-11.91)$ & 0.89 \\
\hline
\end{tabular}

Visceral Patterns in Gastrointestinal Tract Disorders

Dual deficiency of the heart and spleen in females and liver qi depression in males was significantly associated and heart yin deficiency in females was inversely associated with functional dyspepsia. On the other hand, intense heart fire in females and dual deficiency of the heart and spleen in males were significantly associated and liver yin deficiency in females was inversely associated with irritable bowel syndrome. 
Visceral Patterns in Respiratory Disorders

In females, but not in males, some associations between visceral patterns and respiratory disorders were revealed. Phlegm fire harassing the heart was highly significantly associated with hyperventilation syndrome in females. Phlegm turbidity was inversely associated with bronchial asthma.

\section{Visceral Patterns in Other Disorders}

Liver yin deficiency, kidney yin deficiency, and phlegm clouding the pericardium were highly significantly associated with dystonia in females, while phlegm turbidity was inversely associated. Liver yin deficiency was highly significantly associated and yin deficiency was inversely associated with autonomic ataxia in males.

\section{Discussion}

One limitation of the present study is that pulse, tongue, and abdominal examinations were not conducted. Since no standard scoring system for traditional Chinese medicine is available, for the present study, the extent of each pattern had to be tentatively based on the assessment of the chief complaints and the questionnaires. Since the subjects were patients at a tertiary medical care center, the results cannot be generalized. However, the large number of participants with disorders that were more severe and typical for this center enabled the demonstration of many significant associations between relatively rare disorders and patterns.

\section{Patterns in Eating Disorders}

In the present study, liver qi depression, liver fire flaming upward, and phlegm clouding the pericardium in addition to dual deficiency of the heart and spleen were significantly associated with bulimia nervosa in females. Out of these, liver fire flaming upward and phlegm clouding the pericardium result from long-lasting liver qi depression. Liver fire flaming upward is characteristic of irritation. This result is consistent with the fact that irritation tends to result in hyperphagia. Therefore, clearing liver fire would be effective in treating bulimia nervosa.

In the previous study, not only excess patterns but also deficiency patterns were considered for treating bulimia nervosa. Actually, in a randomized crossover pilot study, patients with bulimia nervosa were treated with acupuncture for spleen qi deficiency, stomach heat, heart yin deficiency, or heart qi deficiency in addition to liver qi depression [6]. Dual deficiency of the heart and spleen, a kind of deficiency pattern, was also significantly associated with bulimia nervosa in the present study. Therefore, different diagnosis would be important in this disorder.

In contrast to bulimia nervosa, no pattern was significantly associated with anorexia nervosa. This result indicates that therapy according to traditional Chinese medicine would be more difficult in anorexia nervosa than in bulimia nervosa patients.

\section{Patterns in Somatoform Disorders}

Generally, pain disorder is characteristic of fixed pain. According to the traditional Chinese theory, fixed pain is attributed to blood stasis or water retention. Many Kampo formulas for pain disorder such as goshuyuto and goshakusan contain diuretic remedies. This is consistent with our result that phlegm turbidity was significantly associated with pain disorder in males. In contrast to fixed pain, migratory pain is attributed to liver qi depression. This theory is also consistent with the present study, since liver qi depression was rather 
inversely associated with pain disorder in males. No association was observed between all forms of somatoform disorders and the patterns. This result indicates that somatoform disorder is aggregate of miscellaneous patterns.

\section{Patterns in Primary Insomnia}

In the present study, only liver fire flaming upward was significantly associated with primary insomnia. According to the traditional Chinese theory, it is not only this pattern but also phlegm fire harassing the heart and kidney yin deficiency, in addition to dual deficiency of the heart and spleen that account for insomnia including depression. This is partially consistent with the present study. Out of these patterns, we have previously reported that phlegm fire harassing the heart was significantly associated with depression in females aged $>40$ years [9]. In the present study, depression was excluded to reach a diagnosis of primary insomnia. Therefore, it is supposed that phlegm fire harassing the heart was also excluded from the diagnosis of primary insomnia in the present study.

\section{Patterns in Gastrointestinal Tract Disorders}

In males, dual deficiency of the heart and spleen was significantly associated with irritable bowel syndrome. On the other hand, liver qi depression, which is a kind of excess pattern, was significantly associated with functional dyspepsia. Interestingly, the deficiency-excess pattern in the upper and lower gastrointestinal tract was reverse between males and females.

In females, a kind of deficiency pattern, namely dual deficiency of the heart and spleen, was significantly associated with functional dyspepsia. To the contrary, a kind of excess pattern, intense heart fire, was significantly associated with irritable bowel syndrome. For the diarrhea type of irritable bowel syndrome, the Kampo formula hangeshashinto is applied. This formula contains galenicals that clear the heat below the heart in order to harmonize the stomach yang and the spleen ying. Although gastrointestinal symptoms are not common in intense heart fire, heat in the heart might influence the gastrointestinal tract including the stomach below the heart.

Recently, it has been reported that the rate of overlap between functional dyspepsia and irritable bowel syndrome could be in the range of 11 and 27\% [10]. It is supposed that these disorders have some common mechanisms. This report coincides with the result in the present study that dual deficiency of the heart and spleen was associated with these disorders in both sexes.

\section{Patterns in Respiratory Disorders}

In females, phlegm fire harassing the heart was significantly associated with hyperventilation syndrome. This is consistent with the traditional Chinese theory that coarse respiration results from stagnant qi transforming into fire. We have previously reported that this pattern is also associated with generalized anxiety disorder in females aged $<40$ years [9]. It was also significantly associated with panic disorder in females (data not shown). In a previous study, panic disorder and generalized anxiety disorder with hyperventilation syndrome overlapped each other [11]. It is supposed that these three disorders have a common mechanism.

\section{Patterns in Other Disorders}

Liver yin deficiency and kidney yin deficiency were extremely and significantly associated with dystonia in females. If nourished by liver blood, the kidney can engender kidney essence. In return, the kidney essence nourishes the liver. In other words, liver and kidney nourish each other. Therefore, yin deficiency tends to appear simultaneously in relation to the liver and kidney. In liver yin deficiency, skeletal muscles fail to be nourished and tend to go into spasm and myalgia, which are supposed to correspond to dystonia. According to the 
traditional Chinese theory, emotions tend to get out of order in liver yin deficiency, which is consistent with the results from our previous study that this pattern is associated with depression with suicide feelings [12] and with the results from other previous studies that dystonia is characteristic of aggression, which is a kind of emotional disorder $[13,14]$.

In males, liver yin deficiency was highly and significantly associated with autonomic ataxia. This disorder is not described in DSM-IV and is not a dependent psychiatric entity. If a patient describes symptoms due to autonomic hyperfunction and psychiatric disorders such as somatoform disorder, generalized anxiety disorder, or depressive disorder are ruled out, autonomic ataxia is tentatively diagnosed. In these subjects, myotonia and dizziness were apparent as the chief complaints, consistent with liver yin deficiency. The highly significant association between this disorder and a single pattern observed in the present study indicates that it might be a single entity in contrast to the general recognition that it is diagnosed by exclusion.

This pattern was associated with another disorder, namely dystonia in females as mentioned above. This sexual difference, in addition to the sexual pattern differences in gastrointestinal disorders mentioned above, indicates that different viscera are vulnerable to some patterns according to sex.

The limitation of the present study was that we did not refer to pulse and tongue diagnoses. If these had been included in the calculation, a more accurate evaluation of the patterns would have been possible.

In conclusion, a detailed investigation of the patterns in a large number of patients with psychiatric disorders could reveal specific patterns associated with some disorders. In addition, sex should be taken into account, since some patterns were different between males and females, and the association between some disorders and the patterns existed only in males or females.

\section{Appendix 1}

The Correspondence between Symptoms Described as Chief Complaints or in the KMI Questionnaire and Each Visceral Pattern

\begin{tabular}{|c|c|c|c|c|c|c|c|c|c|c|}
\hline Symptom & $\begin{array}{l}\text { KMI } \\
\text { ques- } \\
\text { tion } \\
\text { number }\end{array}$ & $\begin{array}{l}\text { Liver } \\
\text { qi de- } \\
\text { pres- } \\
\text { sion }\end{array}$ & $\begin{array}{l}\text { Liver } \\
\text { fire } \\
\text { flaming } \\
\text { upward }\end{array}$ & $\begin{array}{l}\text { In- } \\
\text { tense } \\
\text { heart } \\
\text { fire }\end{array}$ & $\begin{array}{l}\text { Phlegm- } \\
\text { fire } \\
\text { harassing } \\
\text { the heart }\end{array}$ & $\begin{array}{l}\text { Phlegm } \\
\text { clouding } \\
\text { the } \\
\text { pericardium }\end{array}$ & $\begin{array}{l}\text { Dual defi- } \\
\text { ciency of } \\
\text { the heart } \\
\text { and spleen }\end{array}$ & $\begin{array}{l}\text { Liver } \\
\text { yin } \\
\text { defi- } \\
\text { ciency }\end{array}$ & $\begin{array}{l}\text { Heart } \\
\text { yin } \\
\text { defi- } \\
\text { ciency }\end{array}$ & $\begin{array}{l}\text { Kidney } \\
\text { yin } \\
\text { defi- } \\
\text { ciency }\end{array}$ \\
\hline Abdominal fullness & & & & & & & $\circ$ & & & \\
\hline Abdominal wind & & $\circ$ & & & & & & & & \\
\hline Abortion & & & & & & & & & & $\mathrm{O}$ \\
\hline Affective lability & & & & & $\mathrm{O}$ & & & & & \\
\hline Afternoon fever & & & & & $\circ$ & & & & $\circ$ & $\circ$ \\
\hline Alopecia & & & & & & & & & & O \\
\hline Alveolar pyorrhea & & & & & & & & & & o \\
\hline Amnesia & & & & & & o & o & & O & \\
\hline Anorexia & C-07 & & & & O & o & o & & & \\
\hline Anxiety & B-19 & & & & & & o & & & \\
\hline Appendicular numbness & & & & & & & & $\circ$ & & \\
\hline Asthenopia & $\mathrm{C}-18$ & & & & & o & & o & & \\
\hline Bitter taste in the mouth & & & o & & & & & & & \\
\hline Breathlessness & & & & & & & & & $\circ$ & \\
\hline Burping & & o & & & & & & & & \\
\hline Cheilitis & & & & $\mathrm{O}$ & & & & & & \\
\hline Chest oppression & & $\mathrm{O}$ & & $\mathrm{O}$ & & & & & o & \\
\hline
\end{tabular}


Kondo et al.: Identification of Visceral Patterns in Patients with Stress-Related Disorders

\begin{tabular}{|c|c|c|c|c|c|c|c|c|c|c|}
\hline Symptom & $\begin{array}{l}\text { KMI } \\
\text { ques- } \\
\text { tion } \\
\text { number }\end{array}$ & $\begin{array}{l}\text { Liver } \\
\text { qi de- } \\
\text { pres- } \\
\text { sion }\end{array}$ & $\begin{array}{l}\text { Liver } \\
\text { fire } \\
\text { flaming } \\
\text { upward }\end{array}$ & $\begin{array}{l}\text { In- } \\
\text { tense } \\
\text { heart } \\
\text { fire }\end{array}$ & $\begin{array}{l}\text { Phlegm- } \\
\text { fire } \\
\text { harassing } \\
\text { the heart }\end{array}$ & $\begin{array}{l}\text { Phlegm } \\
\text { clouding } \\
\text { the } \\
\text { pericardium }\end{array}$ & $\begin{array}{l}\text { Dual defi- } \\
\text { ciency of } \\
\text { the heart } \\
\text { and spleen }\end{array}$ & $\begin{array}{l}\text { Liver } \\
\text { yin } \\
\text { defi- } \\
\text { ciency }\end{array}$ & $\begin{array}{l}\text { Heart } \\
\text { yin } \\
\text { defi- } \\
\text { ciency }\end{array}$ & $\begin{array}{l}\text { Kidney } \\
\text { yin } \\
\text { defi- } \\
\text { ciency }\end{array}$ \\
\hline Compulsion & & & & & & & & & 0 & \\
\hline Conjunctival hyperemia & & & 0 & & 0 & & & & & \\
\hline Constipation & & $\circ$ & $\circ$ & $\circ$ & $\circ$ & & & & & \\
\hline Convulsion & & O & & & & $\circ$ & & $\circ$ & & \\
\hline Cramp & & & & & & & & $\circ$ & & \\
\hline Delayed menstruation & & $\circ$ & & & & & $\circ$ & $\circ$ & & $\mathrm{O}$ \\
\hline Delirium & & & & & $\circ$ & $\circ$ & & & & \\
\hline Dementia & & & & & & $\circ$ & & & & \\
\hline Destructive act & & & & & O & & & & & \\
\hline Diarrhea & & O & & & & & O & & & \\
\hline Difficulty in falling asleep & $\mathrm{C}-44$ & & $\circ$ & $\circ$ & $\circ$ & & O & & 0 & \\
\hline Disruption of sleep & & & $\circ$ & O & $\circ$ & & O & & 0 & \\
\hline Dizziness & $\mathrm{C}-38$ & & $\circ$ & & & & O & $\circ$ & $\circ$ & 0 \\
\hline Early morning awakening & & & $\circ$ & $\circ$ & $\circ$ & & O & & 0 & \\
\hline Easy fatigability & & & & & & 0 & O & & & \\
\hline Epigastric oppression & & & & & & & ○ & & & \\
\hline Excessive dreaming & C-46 & & $\circ$ & 0 & $\circ$ & & 0 & & 0 & \\
\hline Fasciculation & C-43 & O & & & & 0 & & 0 & & \\
\hline $\begin{array}{l}\text { Feeling of heaviness in the } \\
\text { upper limbs }\end{array}$ & & 0 & & & & & & & & \\
\hline Feeling of thoracic heat & & & & 0 & & & & & & \\
\hline
\end{tabular}

Feeling of thoracic heat

Flush

Frequent urination

C-31 O

General fatigue

C-03

Genital bleeding

Gray hair

Headache

Heavy-headed feeling

Hematemesis

Hot flush

Hypacusis

Hyperkinesia

Hyperventilation

Hypobulia

Hyposthenia in the lumbar

part or lower limbs

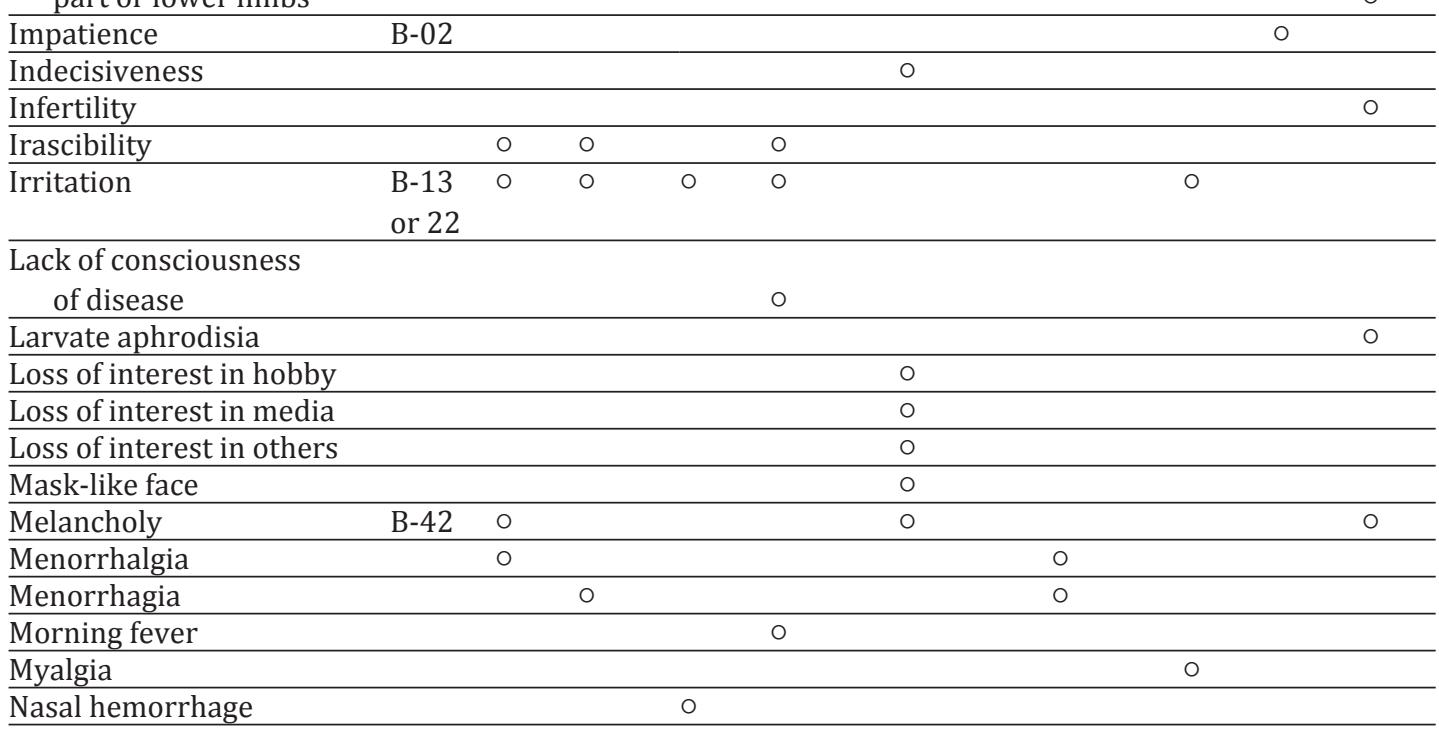


Kondo et al.: Identification of Visceral Patterns in Patients with Stress-Related Disorders

\begin{tabular}{|c|c|c|c|c|c|c|c|c|c|c|}
\hline Symptom & $\begin{array}{l}\text { KMI } \\
\text { ques- } \\
\text { tion } \\
\text { number }\end{array}$ & $\begin{array}{l}\text { Liver } \\
\text { qi de- } \\
\text { pres- } \\
\text { sion }\end{array}$ & $\begin{array}{l}\text { Liver } \\
\text { fire } \\
\text { flaming } \\
\text { upward }\end{array}$ & $\begin{array}{l}\text { In- } \\
\text { tense } \\
\text { heart } \\
\text { fire }\end{array}$ & $\begin{array}{l}\text { Phlegm- } \\
\text { fire } \\
\text { harassing } \\
\text { the heart }\end{array}$ & $\begin{array}{l}\text { Phlegm } \\
\text { clouding } \\
\text { the } \\
\text { pericardium }\end{array}$ & $\begin{array}{l}\text { Dual defi- } \\
\text { ciency of } \\
\text { the heart } \\
\text { and spleen }\end{array}$ & $\begin{array}{l}\text { Liver } \\
\text { yin } \\
\text { defi- } \\
\text { ciency }\end{array}$ & $\begin{array}{l}\text { Heart } \\
\text { yin } \\
\text { defi- } \\
\text { ciency }\end{array}$ & $\begin{array}{l}\text { Kidney } \\
\text { yin } \\
\text { defi- } \\
\text { ciency }\end{array}$ \\
\hline Nausea & & $\circ$ & & & & & $\circ$ & & & \\
\hline Nervosity & A-01 & O & & & & & & & & $\mathrm{O}$ \\
\hline Night sweating & & & & & & & & 0 & 0 & 0 \\
\hline Nightmares & & & & & 0 & & o & & & \\
\hline Ophthalmalgia & & & O & & & & & & & \\
\hline \multicolumn{11}{|l|}{ Pale complexion } \\
\hline Palpitation & C-09 & & & & & & O & & O & \\
\hline Phobia & & & & & & & & & & 0 \\
\hline Pollution & & & & & & & & & & $\mathrm{O}$ \\
\hline Premature menstruation & & & O & & & & O & & & \\
\hline Protracted urination & & O & & & & & & & & \\
\hline Psychomotor depression & & & & & & o & & & & \\
\hline Retardation of thought & $\mathrm{C}-36$ & & & & & O & & & O & \\
\hline Rugitus & & O & & & & & & & & \\
\hline Sadness & & & & & 0 & & & & & \\
\hline Sense of residual urine & & O & & & & & & & & \\
\hline Sexless & & o & & & & & & & & \\
\hline Sigh & & O & & & & & & & & \\
\hline Slight menorrhea & & & & & & & & 0 & & O \\
\hline Sporadic headache & & & O & & & & & & & \\
\hline Sputum & & & & & 0 & O & & & & \\
\hline Stiffness in the shoulders & & O & & & & & & & & \\
\hline Stomatitis & & & & o & 0 & & & & & \\
\hline Systemic convulsion & C-41 & O & & & & O & & $\mathrm{O}$ & & \\
\hline Thirst & $\mathrm{C}-20$ & & o & o & O & & & 0 & 0 & 0 \\
\hline Tinnitus & C-19 & & 0 & & & & & & & 0 \\
\hline $\begin{array}{l}\text { Unpleasant sensation of } \\
\text { laryngopharynx }\end{array}$ & & 0 & & & & & & & & \\
\hline $\begin{array}{l}\text { Vexing heat in the chest, } \\
\text { palms and soles }\end{array}$ & & & & & & & & & o & 0 \\
\hline Vomiting & & o & & & & & $\circ$ & & & \\
\hline Weight loss & C-08 & & & & & & & 0 & 0 & 0 \\
\hline Xerasia & & & & & & & & & & 0 \\
\hline Xeroderma & & & & & & & & 0 & o & 0 \\
\hline
\end{tabular}

\section{References}

1 van der Watt G, Laugharne J, Janca A: Complementary and alternative medicine in the treatment of anxiety and depression. Curr Opin Psychiatry 2008;21:37-42.

2 Mukai M, Baba S, Asao T, Suzuki F, Aoyama H: Traditional Chinese medicine in psychiatry: liver diseases and blood-stagnation (in Japanese). Clin J Trad Chin Med 2002;24:88-90.

3 Mukai M, Baba S, Suzuki F: Traditional Chinese medicine in psychiatry: intense heart fire pattern (in Japanese). Clin J Trad Chin Med 2002;23:70-73.

4 Mukai M, Baba S, Asao T, Suzuki F, Aoyama H, Fujii K: Traditional Chinese medicine in psychiatry: deficiency pattern (in Japanese). Clin J Trad Chin Med 2002;24:78-80.

5 Mukai M, Baba S, Suzuki F: Traditional Chinese medicine in psychiatry: pattern of phlegm-fire harassing the heart and phlegm turbidity (in Japanese). Clin J Trad Chin Med 2002;23:72-75.

6 Fogarty S, Harris D, Zaslawski C, McAinch AJ, Stojanovska L: Acupuncture as an adjunct therapy in the treatment of eating disorders: a randomised cross-over pilot study. Complement Ther Med 2010;18:233-240.

7 Matsuoka Y: KMI (in Japanese); in Kawano T, Suematsu H, Arazato R (eds): Psychological Test for Psychosomatic Medicine (Shinshinigakuno tameno shinritesuto). Tokyo, Asakura Publications, 1990, pp 45-49.

8 WHO Regional Office for the Western Pacific: WHO international standard terminologies on traditional medicine in the Western Pacific region. Manila, WPRO Nonserial Publication, 2007. 
9 Kondo T, Yoshimasu K, Tokunaga S, Sugahara H, Kanemitsu Y, Kubo C: Age-related changes in visceral patterns associated with anxiety disorders or depression according to traditional Chinese medicine. Integr Med Int 2014;1:80-92.

10 Suzuki H, Hibi T: Overlap syndrome of functional dyspepsia and irritable bowel syndrome - are both diseases mutually exclusive? J Neurogastroenterol Motil 2010;17:360-365.

11 de Ruiter C, Garssen B, Rijken H, Kraaimaat F: The hyperventilation syndrome in panic disorder, agoraphobia and generalized anxiety disorder. Behav Res Ther 1989;27:447-452.

12 Kondo T, Yanagida M, Kame S: Traditional Chinese patterns in psychosomatic and psychiatric medicine. Int J Integr Med 2013;1:81-94.

13 Sugahara H: Psychosomatic point of view; in Kayase H (ed): Practical Therapy for Spasmodic Torticollis at Each Department. Tokyo, Shinko Medical Publishing, 2002, pp 61-92.

14 Schulz G, Hemke S: Torticollis spasmodicus. A contribution to psychogenesis and psychotherapy (in German). Psychiatr Neurol Med Psychol (Leipz) 1988;40:564-571. 